OPEN ACCESS

Edited by:

Yan Guo,

China Agricultural University, China

Reviewed by:

Xiuli Hu,

Henan Agricultural University, China

Yangrong Cao,

Huazhong Agricultural University,

China

${ }^{*}$ Correspondence:

Zhao-Shi Xu

xuzhaoshi@caas.cn

You-Zhi Ma

mayouzhi@caas.cn

${ }^{t}$ These authors have contributed equally to this work

${ }^{\ddagger}$ Present address:

Li-Juan Yin,

Agricultural Genomics Institute

at Shenzhen, Chinese Academy

of Agricultural Sciences,

Shenzhen, China

Specialty section:

This article was submitted to

Plant Abiotic Stress,

a section of the journal

Frontiers in Plant Science

Received: 22 November 2018

Accepted: 04 July 2019

Published: 23 July 2019

Citation:

Zhao M-J, Yin L-J, Ma J, Zheng J-C, Wang $Y-X$, Lan J-H,

Fu J-D, Chen M, Xu Z-S and Ma Y-Z

(2019) The Roles of GmERF135

in Improving Salt Tolerance

and Decreasing ABA Sensitivity

in Soybean. Front. Plant Sci. 10:940.

doi: 10.3389/fp/s.2019.00940

\section{The Roles of GmERF135 in Improving Salt Tolerance and Decreasing ABA Sensitivity in Soybean}

\author{
Meng-Jie Zhao't, Li-Juan Yin'tł, Jian Ma't, Jia-Cheng Zheng ${ }^{3}$, Yan-Xia Wang4, \\ Jin-Hao Lan ${ }^{5}$, Jin-Dong Fu', Ming Chen', Zhao-Shi Xu' ${ }^{1,2,3 *}$ and You-Zhi Ma ${ }^{1 *}$
}

${ }^{1}$ Institute of Crop Science, Chinese Academy of Agricultural Sciences (CAAS)/National Key Facility for Crop Gene Resources and Genetic Improvement, Key Laboratory of Biology and Genetic Improvement of Triticeae Crops, Ministry of Agriculture, Beijing, China, ${ }^{2}$ Department of Agronomy, Jilin Agricultural University, Changchun, China, ${ }^{3}$ College of Agriculture, Anhui University of Science and Technology, Fengyang County, China, ${ }^{4}$ Hebei Academy of Agriculture and Forestry Sciences, Research Center of Wheat Engineering Technology of Hebei, Shijiazhuang, China, ${ }^{5}$ College of Agronomy and Plant Protection, Qingdao Agricultural University, Qingdao, China

Abscisic acid (ABA) mediates various abiotic stress responses, and ethylene responsive factors (ERFs) play vital role in resisting stresses, but the interaction of these molecular mechanisms remains elusive. In this study, we identified an ABA-induced soybean ERF gene GmERF135 that was highly up-regulated by ethylene (ET), drought, salt, and low temperature treatments. Subcellular localization assay showed that the GmERF135 protein was targeted to the nucleus. Promoter cis-acting elements analysis suggested that numerous potential stress responsive cis-elements were distributed in the promoter region of GmERF135, including ABA-, light-, ET-, gibberellin (GA)-, and methyl jasmonate (MeJA)-responsive elements. Overexpression of GmERF135 in Arabidopsis enhanced tolerance to drought and salt conditions. In addition, GmERF135 promoted the growth of transgenic hairy roots under salt and exogenous ABA conditions. These results suggest that soybean GmERF135 may participate in both ABA and ET signaling pathways to regulate the responses to multiple stresses.

Keywords: ABA, ethylene-responsive factor, hypocotyl elongation, root growth, response mechanism, salt tolerance, soybean

\section{INTRODUCTION}

As a vital hormone in plants, abscisic acid (ABA) is essential to a myriad of aspects of plant growth and developmental processes, including plant embryo development, seed maturation, fruit maturity, and stomatal movement (Finkelstein et al., 2002). ABA signal transduction has been studied for many years (Pandey et al., 2006, 2009; Shen et al., 2006; Ma et al., 2009; Park et al., 2009; Yang and Guo, 2018), and the widely accepted molecular mechanism is that the pyrabactin resistant/PYR-like/regulatory component of ABA receptor (PYR/PYL/RCAR) acts as an ABA receptor which can bind to $A B A$, and then binds to and inhibits protein phosphatases type $2 \mathrm{C}$ (PP2Cs) (Santiago et al., 2009). In addition, the activity of SNF1-related protein kinases 2 (SnRK2s) is enhanced and can phosphorylate $\mathrm{ABRE}$ binding factors (AREB/ABFs) to induce physiological and biochemical changes in the process of ABA response (Hubbard et al., 2010; Yoshida et al., 2015). A recent study showed that ABFs can directly bind to the promoters of group A PP2C genes, and 
rapidly induce their expression on exogenous ABA treatments (Wang et al., 2018). However, the downstream molecular mechanism is yet not clearly understood.

Many transcription factors, such as MYC/MYB, bZIP/ABRE, $\mathrm{AP} 2 / \mathrm{ERF}$, and NAC, are regulated by ABA. The AP2/ERF family, the largest plant transcription factor family (Okamuro et al., 1997), can be divided into three subfamilies: the AP2, ERF, and RAV subfamilies. Among them, the ERF subfamily can specifically bind to GCC-box and/or the dehydrationresponsive element/C-repeat (DRE/CRT) cis-acting elements (Allen et al., 1998).

The AP2/ERF family is involved in responses to various abiotic stresses and exogenous hormones (Xu et al., 2008, 2011; Klay et al., 2018). For example, overexpressing TaERF1 enhances tolerance of physiological and environmental stresses, such as salt, drought, low temperature, exogenous $\mathrm{ABA}$, ethylene (ET), salicylic acid (SA), and disease (Xu et al., 2007). Transgenic rice plants expressing OsERF922 displayed higher susceptibility to Magnaporthe oryzae and $\mathrm{NaCl}$ compared to the wild type (WT), while the knockout mutant and RNAi lines enhanced resistance to these stresses (Liu et al., 2012). A recent study described how ABA modulates the expression level of ERF family, showing that the expression levels of ABA-responsive genes such as RD22, $L E A 3$, and $P O D s$ were up-regulated after overexpressing OsERF101 in rice, which enhanced its susceptibility to ABA (Jin et al., 2018).

Transcriptomic analysis of grapevine organs treated with or without ABA showed that ERF members were involved in differently expressed genes (DEGs), and the ERF subfamily had the most significant change compared to other transcription factors (Rattanakon et al., 2016). Although the ABA signal pathway has been extensively studied, it is yet unclear how regulation of the expression of downstream genes via ERF subfamily could enhance abiotic stress tolerances in soybean. To investigate whether the ERF subfamily is modulated by the ABA signal in soybean, we studied the response to exogenous $\mathrm{ABA}$ and identified the functions of GmERF135 in transgenic Arabidopsis and soybean hairy roots.

\section{MATERIALS AND METHODS}

\section{Plant Materials and Treatment}

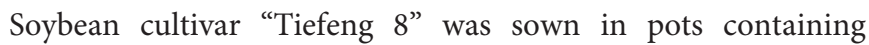
vermiculite and grown at $25^{\circ} \mathrm{C}$ for 14 days and then treated with various abiotic stresses. For the various treatments, soybean plants were exposed in the air for rapid drought, dipped into $200 \mathrm{mM} \mathrm{NaCl}$ for salt stress, placed in $42^{\circ} \mathrm{C} / 4^{\circ} \mathrm{C}$ chamber for high/low temperature stress, respectively. They were also placed in an airtight container filled with ET, or dipped in $100 \mu \mathrm{M}$ ABA, $50 \mu \mathrm{M}$ salicylic acid (SA), or $50 \mu \mathrm{M}$ jasmonate (JA) for exogenous hormone stresses. Leaves of these plants were collected after $0,0.5,1,2,5,12$, and $24 \mathrm{~h}$ treatment and then immediately stored at $-80^{\circ} \mathrm{C}$ for RNA extraction.

\section{Gene Structure and Protein Domain of ERFs in Soybean}

The whole genome data of the candidate ERF genes was obtained from JGI Glyma1.0 annotation ${ }^{1}$ (Goodstein et al., 2012). Gene structure was analyzed by submitting the CDS of the candidate genes and whole genomic DNA sequences to the Gene Structure Display Server (GSDS) website ${ }^{2}$. Protein Fold Recognition Server (PHYRE2) ${ }^{3}$ was used for analysis of structural homology modeling of these genes, and DOG 2.0 was used to draw the protein domains.

\section{Quantitative Real-Time PCR (qRT-PCR)}

Total RNA of the soybean plants was extracted using the TRIzol reagent (Invitrogen, Carlsbad, CA, United States). The specific primer pairs for the 16 genes were designed by Primer Premier 5.0 according to the cDNAs. qRT-PCR was conducted using the SYBR Premix Ex Taq ${ }^{\text {TM }}$ kit (TAKARA, Kusatsu, Japan) and the ABI Prism 7500 real-time PCR system (Thermo Fisher Scientific, Waltham, MA, United States). The $2^{-\Delta \Delta}$ CT method was used to conduct qRT-PCR analysis (Le et al., 2011). Soybean Actin (U60506) was used as an internal control and sequence of specific primers were shown in Supplementary Table S1.

\section{Isolation and Promoter Analysis of GmERF135}

The GmERF135 gene was amplified by PCR and the primers for cloning the GmERF135 were $5^{\prime}$ AATCATTATGTGTGGCGGTGCC- $3^{\prime}$ and $5^{\prime}$-TATTCCTCGC TAATCGAAACTCCAGAG- $3^{\prime}$. The PCR product was then cloned into the pEASY-T1 vector (TransGen, Beijing, China). 1,886 bp promotor region of GmERF135 was cloned and submitted to PLACE ${ }^{4}$ and PlantCARE ${ }^{5}$ databases to analyze the putative cis-acting elements in the promoter region (Lescot et al., 2002).

\section{Subcellular Localization Analysis}

The cDNA of GmERF135 were augmented with PCR, connected to the $\mathrm{N}$-terminus of humanized green fluorescent protein ( $h G F P$ ) reporter gene under the control of the double Cauliflower Mosaic Virus $(2 \times$ CaMV $) 35 S$ promoter, and a recombinant plasmid was obtained (Scott et al., 1999). The recombinant plasmid was introduced into onion epidermal cells, while the onion epidermal cells with $h G F P$ vector acted as the control. Fluorescence microscopy was used to identify $h G F P$ expression (Xu et al., 2007; He et al., 2016).

\section{Generation of Transgenic Arabidopsis and Stress Treatments}

AT5G47230 is the orthologue of GmERF135 in Arabidopsis, which was named ERF2 and used to investigate function in

\footnotetext{
${ }^{1}$ https://phytozome.jgi.doe.gov/pz/portal.html

${ }^{2} \mathrm{http}: / /$ gsds.cbi.pku.edu.cn/

${ }^{3}$ http://www.sbg.bio.ic.ac.uk/phyre2/html/page.cgi?id=index

${ }^{4}$ http://www.dna.affrc.go.jp/PLACE/

${ }^{5} \mathrm{http} / / /$ bioinformatics.psb.ugent.be/webtools/plantcare/html/
} 
responses to various stresses. The seeds of two mutants, erf21 and erf2-2 (SALK_126889, SALK_076967) were mutated via T-DNA insertion.

The cDNA of GmERF135 was obtained by using the specific primer pairs: 5'-TGATTACGCCAAGCTTATGTGTG GCGGTGCC-3', $5^{\prime}$-CCGGGGATCCTCTAGAATCGAAACTC CAGAG-3', and then were cloned into pBI121 under the control of the CaMV $35 \mathrm{~S}$ promoter. The recombinant plasmid 35S::GmERF135 was sequenced and transformed into wild-type (WT) and the two mutants Arabidopsis lines using the vacuum infiltration method (Bechtold and Pelletier, 1998; Liu et al., 2013). T3 seeds of transgenic lines were selected for further analysis.

For root length assay, 30 seeds of each line were sown on 1/2strength MS growth medium with or without 6\% PEG, $75 \mathrm{mM}$ $\mathrm{NaCl}, 1$-aminocyclopropane-1-carboxylic acid (ACC), or in dark treatment for growth, respectively. At least 30 seedlings per line were randomly selected to measure root length. The cotyledon pieces of each line were recorded every $12 \mathrm{~h}$. Each treatment contained three independent replicates.

\section{Soybean Hairy Root Induction and Stress Treatments}

The Superroot of Lotus corniculatus and Cucumopine-type Agrobacterium rhizogene strain K599 with pGFPGUSPlus were provided by Professor Tian-Fu Han (CAAS, China). Seedling growth, rooting, hairy root induction, and hairy root transformation were performed as described by Chen et al. $(2004,2014)$

GFP positive $\left(\mathrm{GFP}^{+}\right)$hairy roots were induced by $\mathrm{K} 599$ carrying the pGFPGUSPlus-GmERF135 binary vector. These hairy roots were cultured on 1/2 MS medium supplemented with $0,50,85,120$, or $150 \mathrm{mM} \mathrm{NaCl}$ for salt treatment, and 50,100 , or $150 \mu \mathrm{M}$ ABA for hormone treatment, respectively. They were then incubated at $24^{\circ} \mathrm{C}$ under a $16 / 8 \mathrm{~h}$ light/dark cycle condition for 2 weeks. After $24 \mathrm{~h}$ incubation at $105^{\circ} \mathrm{C}$, the increase in dry weight (30 roots per unit) was measured and recorded. Each treatment contained three independent replicates.

\section{RESULTS}

\section{Molecular Characterization of Soybean Targeted ERF Genes}

In a previous study, 160 non-redundant soybean ERFs were identified using the Pfam database and SMART program, and these soybean ERFs were clustered into eight groups (Supplementary Table S2; Zhao et al., unpublished). To comprehensively understand the responses of soybean ERFs to ABA, 16 ERF genes were selected for further investigation according to the phylogenetic tree. Gene structure analysis showed that six genes had no introns, including GmERF106, GmERF132, GmERF135, GmERF41, GmERF49, and GmERF84 (Figure 1). The remaining $10 \mathrm{ERF}$ genes contained one intron, which was distributed in each cDNA region except for GmERF103, GmERF111, and GmERF15. The protein domains of each soybean ERF gene were drawn by DOG2.0 (Figure 2). All the proteins contained a conservative AP2/ERF domain, which was distributed in different positions of each protein sequence. The AP2/ERF domain of the proteins in each group displayed similar locations such as Group I, III, IV, V, VII, and VIII.

An expression pattern map of soybean ERFs was drawn based on the gene-chip data downloaded from the soybean genome database $^{6}$. As shown in Supplementary Figure S1A, the soybean ERFs were expressed at different levels in various tissues and organs. Among them, GmERF111 and GmERF135 showed high expression levels in almost all tissues and organs. GmERF75 showed high expression levels in root and nodule, and GmERF49 was highly expressed in nodule and 10 DAF pod shell. GmERF15 mainly expressed in young leaf. All the candidate genes had different expression patterns.

\section{Isolation and Subcellular Localization Characterization of GmERF135}

Abscisic acid plays an important role in plant growth and development which is closely related to quality and yield in plants. To investigate how ABA affected the expression pattern of the 16 soybean ERF genes, qRT-PCR was conducted. Almost all the ERFs were up-regulated by ABA, except for GmERF49 (Figure 3). The ERF gene GmERF135 was the highest expressed gene after exogenous ABA treatment except for GmERF75 which has been studied (Zhao et al., unpublished). The transcript level of GmERF135 was activated and showed a 17-fold increase within $2 \mathrm{~h}$ after treatment and then declined to normal. Considering the high expression level and ABA-responsive increase in plants, GmERF135 was selected for further study.

The subcellular localization assay was conducted to provide clues to understand intrinsic characteristics of cell activities. $h G F P$ reporter gene fused to C-terminus of GmERF135, which could fluoresce when laser irradiated. The GmERF135::hGFP fused protein only fluoresced in nucleus, while the control fluoresced throughout the entire cell (Figure 4). This result showed that GmERF135 functions in nucleus.

\section{GmERF135 Promoter Region Comprises Diverse Stress-Responsive Elements}

The promoter region is an important part of the gene which could regulate gene expression and control gene action. To investigate the potential regulation mechanism, the promoter region of GmERF135, which has 1886 bp length upstream of the start codon, was isolated. The PLACE and PlantCARE databases were used to analyze the putative regulatory elements in the promoter region. Several regulatory elements were identified to be involved in responses to abiotic stresses and plant hormones (Table 1).

The GmERF135 promoter region contains many ABA and stress responsive elements, including ABA-responsive elements (ABREs, 3 hits), MYBST1 core sequences (4 hits), MYB binding sites (MBS, 1 hit), and DPBF binding sites (2 hits) (Table 1). Except for ABREs, three hormone-responsive elements were predicted, including an ethylene- responsive element (ERE, 1 hit), MeJA-responsive element TGACGmotif (1 hit) and CGTCA-motif (1 hit), and GA-responsive

\footnotetext{
${ }^{6}$ https://soybase.org/soyseq
} 


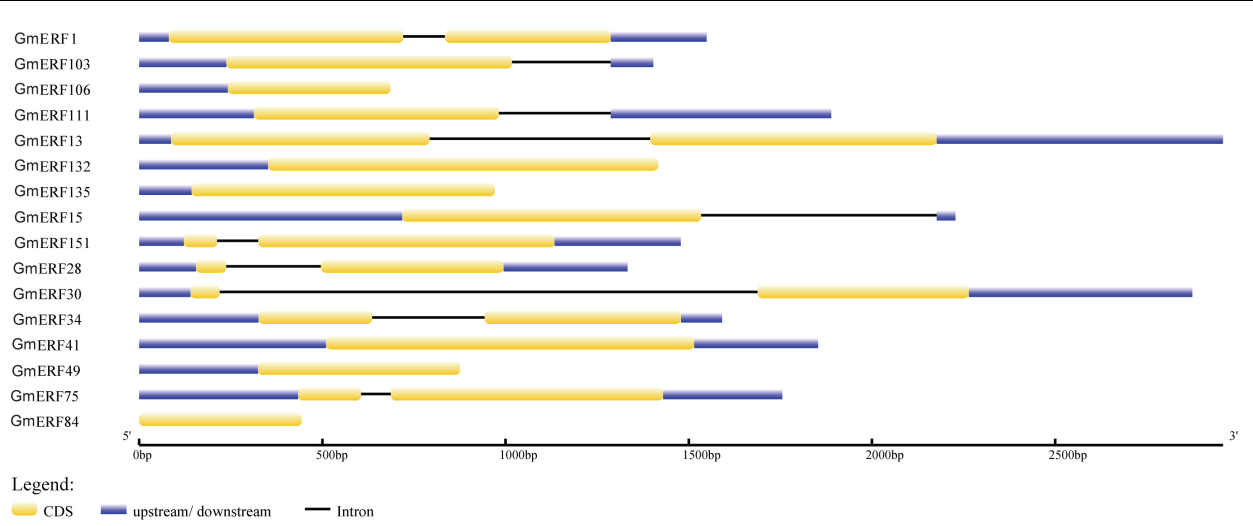

FIGURE 1 | Intron-exon structure of candidate soybean ERF genes. Genome data for candidate ERF genes was obtained from JGI Glyma1.0 annotation. Intron-exon structure was produced using the GSDS online tool by submitting CDSs and genomic sequences of candidate genes. Yellow boxes represent exons, blue boxes represent introns, and black lines represent untranslated regions (UTRs).

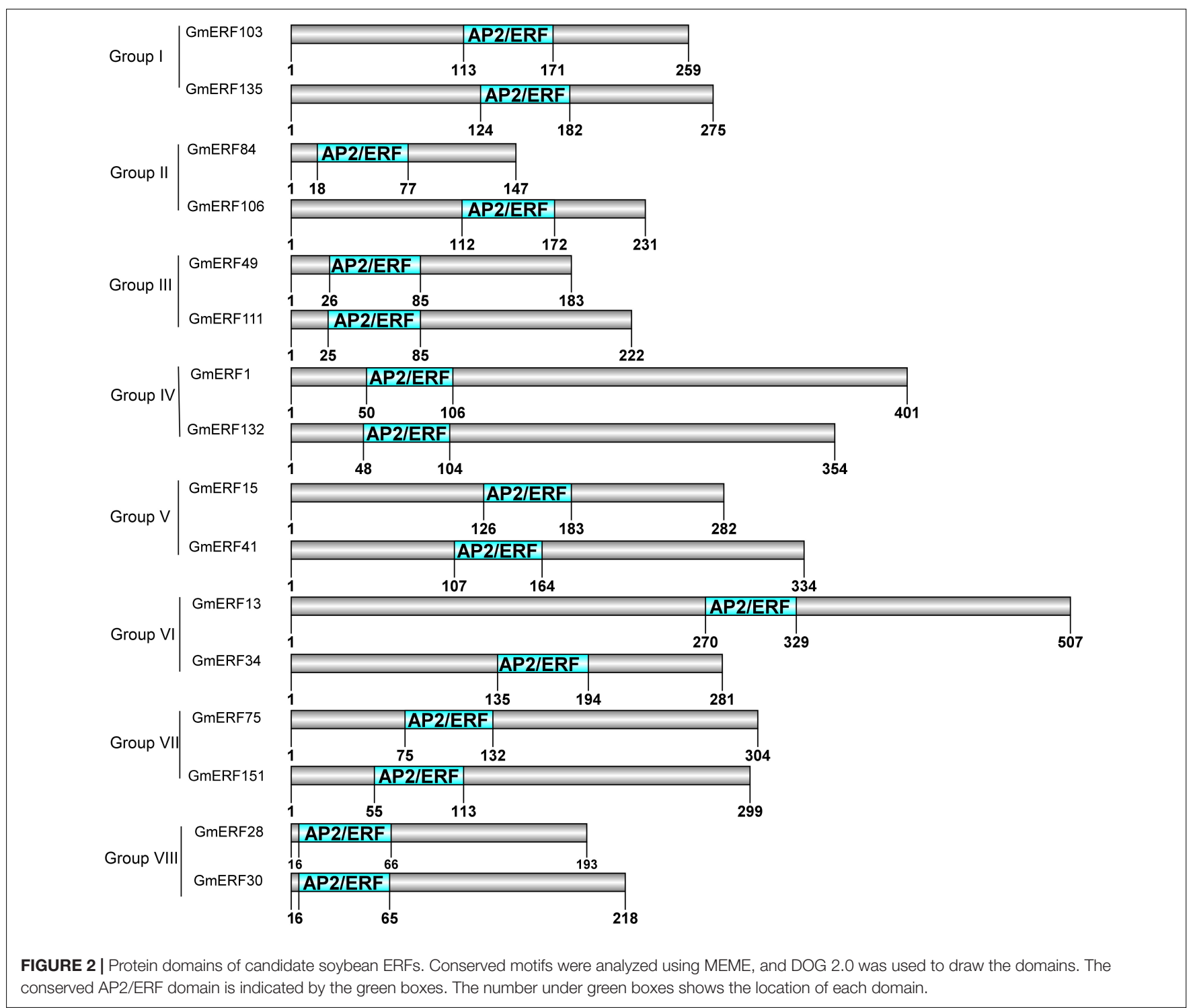



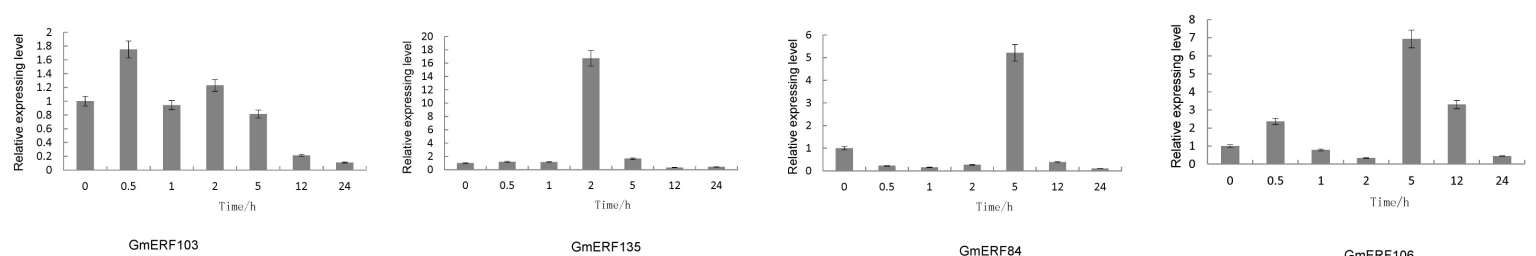

MERF84

GmERF106
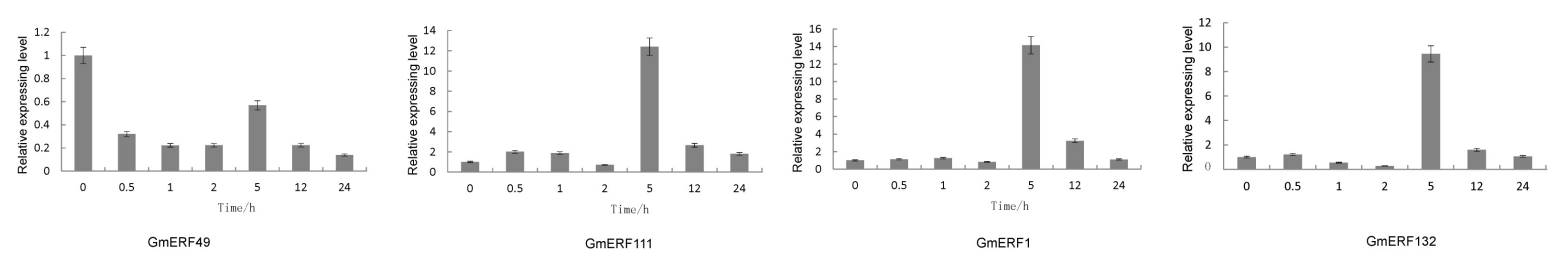

GmERF1

GmERF132
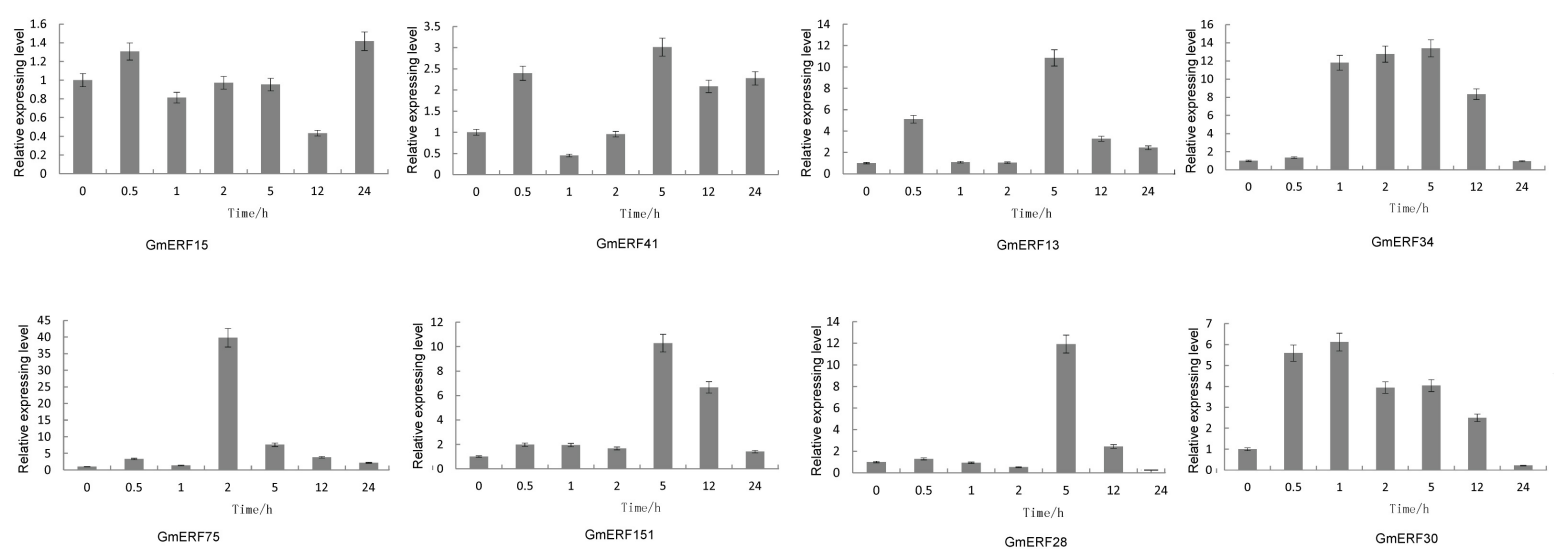

FIGURE 3 | Relative expression levels of ERF genes after ABA treatment. Total RNA was extracted from soybean seedlings after ABA treatment for 0, 0.5, 1, 2, 5, 12 , and $24 \mathrm{~h}$ and used for $\mathrm{QRT}$-PCR. The data were shown as the means \pm SD obtaining from three biological replicates.

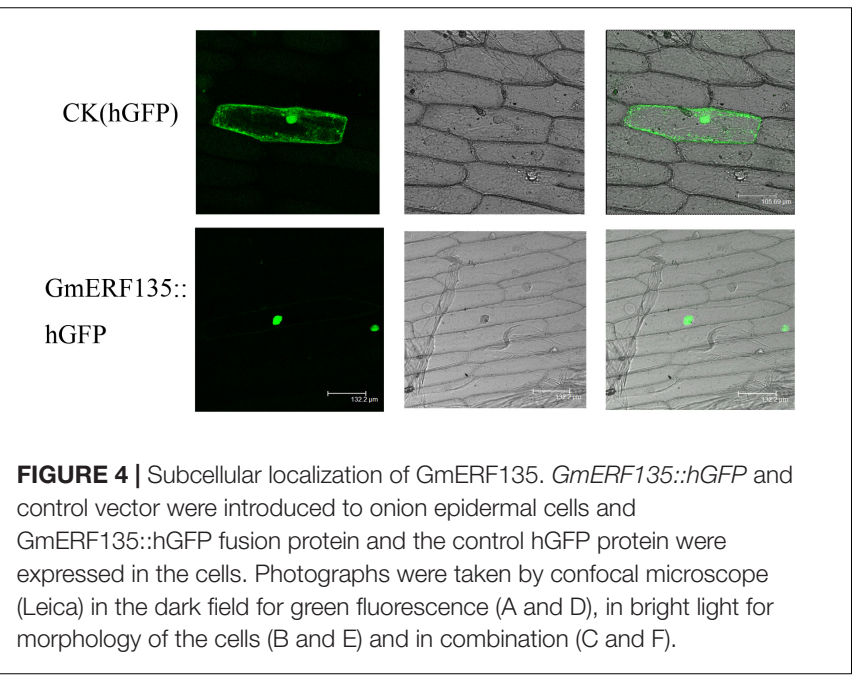

element (GARE, 2 hits). In addition, TCT-motif (1 hit), G-box (2 hits), and Box 4 (1 hit) were found, which could act as light-responsive elements in the promoter region of
GmERF135. These elements suggested that GmERF135 may be involved in responses to multiple abiotic stresses and exogenous hormones.

\section{The Roles of GmERF135 in Responding to Multiple Stimuli}

Quantitative Real-Time PCR was conducted to investigate the expression level of GmERF135 under abiotic stresses, such as drought, salt, high/low temperature, and exogenous hormones including ET, SA, and JA. GmERF135 was induced by almost all the abiotic stresses and exogenous hormones. Remarkably, the expression level of the gene was extremely up-regulated by drought, low temperature, and ET treatment. The transcription of GmERF135 peaked at $5 \mathrm{~h}$ under drought or low-temperature treatment which had 18-fold / 22-fold increases, respectively (Figure 5). The peak of GmERF135 transcription appeared at $12 \mathrm{~h}$ after treatment with ET, which showed a 28-fold increase. In contrast, GmERF135 rapidly reached the maximum transcript level within $0.5 \mathrm{~h}$ under the $\mathrm{NaCl}$ and JA treatments, and then declined to baseline after $1 \mathrm{~h}$. Under the SA treatment, GmERF135 was 
TABLE 1 | Analysis of putative cis-acting elements of GmERF135 promoter.

\begin{tabular}{|c|c|c|c|c|c|}
\hline Cis-acting element & Position & Strand & Matrix score & Sequences & Function \\
\hline ABRE & 747 & + & 6 & CACGTG & ABA responsive element \\
\hline ABRE & 1221 & - & 5 & ACGTG & ABA responsive element \\
\hline ABRE & 748 & + & 5 & ACGTG & ABA responsive element \\
\hline Box 4 & 175 & + & 6 & ATTAAT & Light responsive element \\
\hline CGTCA-motif & 1022 & + & 5 & CGTCA & MeJA-responsive element \\
\hline G-Box & 747 & + & 6 & CACGTG & Light responsive element \\
\hline G-Box & 1221 & + & 6 & CACGTT & Light responsive element \\
\hline MBS & 1295 & + & 6 & CAACTG & ABA and stress responsive element \\
\hline TCT-motif & 201 & + & 6 & TCTTAC & light responsive element \\
\hline TGACG-motif & 1022 & - & 5 & TGACG & MeJA responsive element \\
\hline Core of MYBST1 & 57 & - & 5 & GGATA & ABA and stress responsive element \\
\hline Core of MYBST1 & 532 & - & 5 & GGATA & ABA and stress responsive element \\
\hline Core of MYBST1 & 1725 & + & 5 & GGATA & ABA and stress responsive element \\
\hline Core of MYBST1 & 1818 & - & 5 & GGATA & ABA and stress responsive element \\
\hline DPBF binding site & 108 & + & 7 & ACACNNG & ABA responsive element \\
\hline DPBF binding site & 134 & + & 7 & ACACNNG & ABA responsive element \\
\hline GARE & 1,207 & + & 7 & TAACAAR & Gibberellin responsive element \\
\hline GARE & 654 & + & 7 & TAACAAR & Gibberellin responsive element \\
\hline ERE & 151 & + & 8 & ATाTCAAA & Ethylene responsive element \\
\hline
\end{tabular}

induced and reached the peak within $2 \mathrm{~h}$ (about 7 -fold) and then declined to normal level in $24 \mathrm{~h}$. These results showed that GmERF135 may be involved in responses to multiple stimuli.

\section{GmERF135 Rescued Two erf2 Mutants and Affected Growth of the Root and Cotyledon}

Total RNA was extracted for semi-quantitative PCR from hypocotyls, root, stem, and leaf tissues of normal growth soybean seedlings. Actin primers were used as a parallel reaction to normalize the added template amounts. GmERF135 was predominantly expressed in the leaf, less in the hypocotyl and stem, and very little in the root (Supplementary Figure S1B).

To assess whether GmERF135 could rescue the two mutants, the seeds of transgenic GmERF135::erf2 lines, WT, and two erf2 mutants were sown on the $1 / 2 \mathrm{MS}$ medium for growth and the plant growth rate was recorded each $12 \mathrm{~h}$. Six day later, the phenotypes were imaged (Figures 6A,B).

Growth rate assay showed GmERF135::erf2 lines displayed faster growth rate compared to the mutants (Figure 6C). WT plants required $2 \mathrm{~d}$ of growth to sprout the two pieces of the cotyledon while the two mutants required 4 days. After introduction of GmERF135 in erf2 lines, 3 days was required for the same process. The two mutants needed 6 days to reach 4-leaf stage, while the transgenic GmERF135::erf2 lines had similar growth rate with WT, which only needed $5 \mathrm{~d}$. Root length assay showed there is an approximately 25\% decrease in erf2 mutants compared to WT lines (Figure 6D). After overexpressing GmERF135 in the erf2 mutants, the lines had similar root lengths to WT and the phenotype of which was rescued. These results showed that overexpression of GmERF135 in the two erf2 mutants partly rescued two erf2 mutants and affected growth of root and cotyledon in Arabidopsis.

\section{GmERF135 Promotes Plant Growth Under Drought and Salt Stresses in Arabidopsis}

To determine whether GmERF135 confers abiotic stress tolerance to Arabidopsis plants, 3-day-old WT, erf2 mutants, and GmERF135 overexpression seedlings were transferred to $1 / 2$ MS medium with or without 6\% PEG, $75 \mathrm{mM} \mathrm{NaCl}$, ACC, and dark environment grown for 3 days. The growth rate and the root length of 30 seedlings in each line were recorded (Figures $\mathbf{7 B}, \mathbf{C}$ ). Statistics showed that the average root length of erf2 was shorter than the WT and transgenic Arabidopsis plants under all conditions except for ACC treatment (Figures 7A,B). The transgenic plants displayed larger cotyledon than the WT and mutants under $\mathrm{NaCl}$ and PEG treatments, which suggested that GmERF135 enhanced the growth rate of Arabidopsis plants. Overexpression of GmERF135 in Arabidopsis resulted in a longer root compared to the WT and two erf2 mutants under 6\% PEG, $75 \mathrm{mM} \mathrm{NaCl}$, and dark conditions. No detectable difference was observed between the transgenic lines and WT under ACC conditions.

To further understood the response to various stresses in Arabidopsis, some marker genes, such as NCED3 (Ruggiero et al., 2004), RD29A (Yamaguchi-Shinozaki and Shinozaki, 1994), COR15A (Xu et al., 2011), DREB2A (Liu et al., 1998), ABA1, ABA2 (He et al., 2016), ACO4, and ACS2 (Kim et al., 2013) were selected for qRT-PCR (Supplementary Figure S2). 

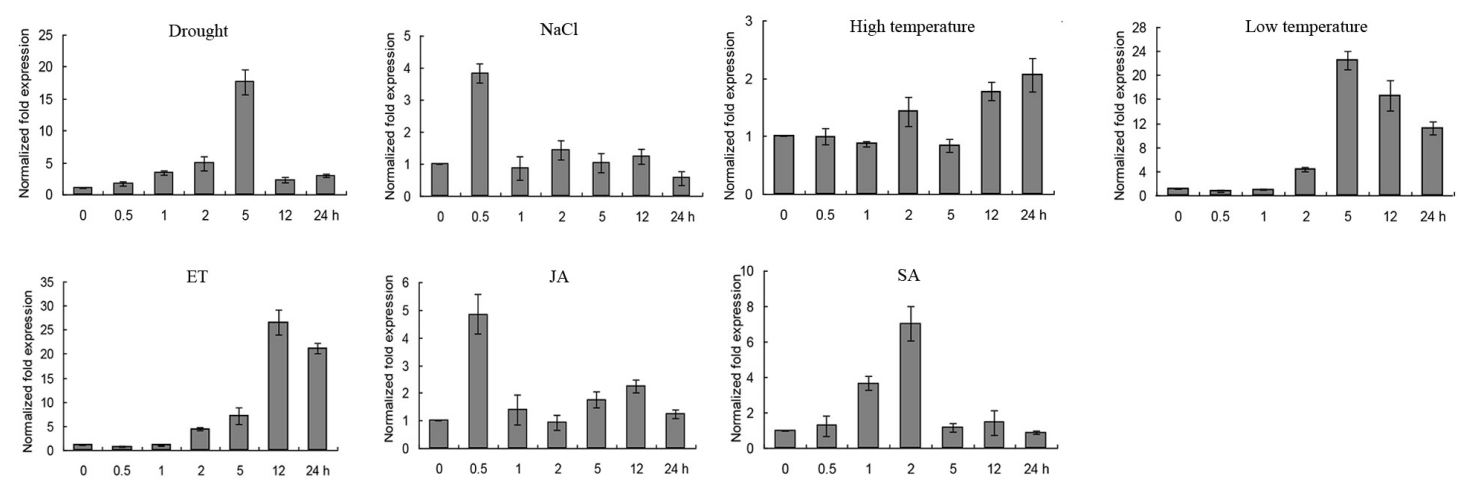

FIGURE 5 | Expression patterns of GmERF135 under multiple stimuli. Two-week soybean seedlings were given various abiotic stresses and exogenous hormones for $0,0.5,1,2,5,12$, and $24 \mathrm{~h}$, which were used to extract RNA and obtained cDNA. The transcript levels of GmERF135 after multiple stresses were quantified by qRT-PCR, including drought, NaCl, high/low temperature, ET (ethylene), JA (jasmonate), and SA (salicylic acid). Data were shown referring to three biological replicates.
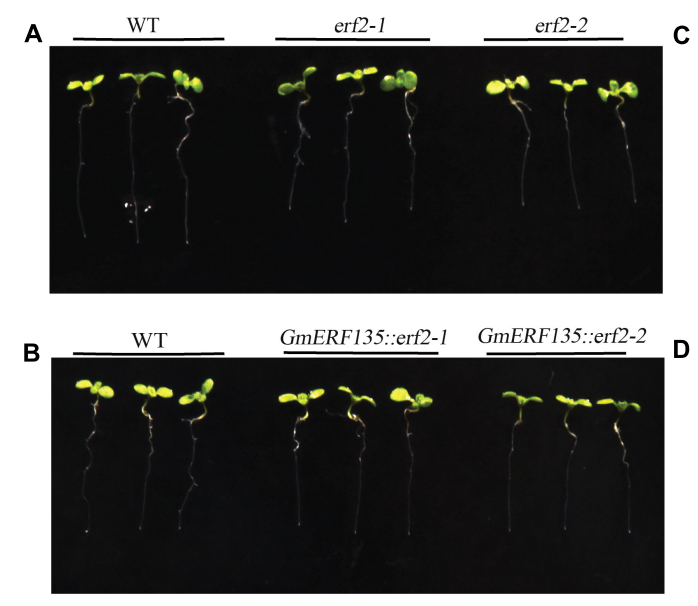

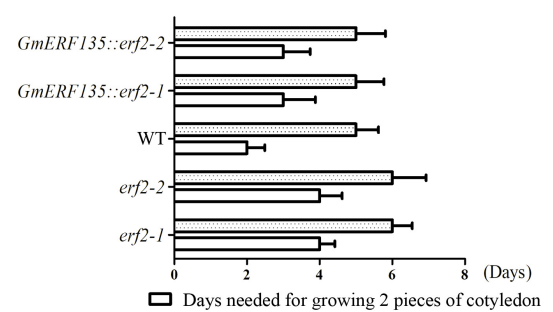

Days needed for growing 2 pieces of cotyledon

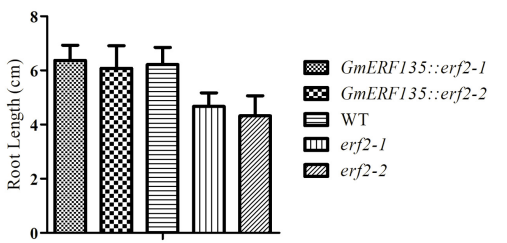

FIGURE 6 | GmERF135 rescued the speed of cotyledon growth in the two mutants. (A) Phenotype of two erf2 mutants and WT Arabidopsis grown on the MS solid medium. (B) Phenotype of two GmERF135::erf2 lines and WT Arabidopsis grown on 1/2 MS solid medium. (C) Days needed for growing the cotyledon. Statistic data were recorded every $12 \mathrm{~h}$. Three independent experiments (20 plants each) were performed. Error bars indicate SD of three biological replicates $(n=3)$. (D) Average root lengths of WT, erf2, and GmERF135::erf2 after grown on 1/2 MS medium for 6 days. Data were shown as the means \pm SD of three biological replicates (at least 30 individuals per line).

\section{GmERF135 Improves Tolerance of Salt and ABA in Soybean}

To investigate the roles of GmERF135 in soybean, the pGUSGmERF135 expression vector was constructed and transformed into soybean hairy roots, which were studied on MS basal medium supplemented with different concentrations of $\mathrm{ABA}$ and $\mathrm{NaCl}$. GmERF135 transgenic soybean hairy roots experienced greater growth under the treatments compared to the vector control, especially under the $100 \mu \mathrm{M} \mathrm{ABA}$ and $85 \mathrm{mM} \mathrm{NaCl}$ conditions (Figure 8A).

Dry weight measurement results showed that GmERF135 hairy roots increased by about $10 \%$ compared to the vector under the MS0 condition, while transgenic hairy roots displayed a $6.24-75 \%$ improvement compared to WT under $\mathrm{NaCl}$ and exogenous $\mathrm{ABA}$ at the different concentrations (Figure 8B). For $\mathrm{NaCl}$ treatment, there has a $6.24-75.03 \%$ increase in the average dry weight of hairy roots overexpressing GmERF135. For exogenous ABA, the transgenic hairy roots displayed a 36$65 \%$ improvement compared to vector control under exogenous ABA treatment at different concentrations (Figure 8B). It is noteworthy that a total of $64.46 \%$ improvement in GmERF135 hairy roots was observed under the $100 \mu \mathrm{M} \mathrm{ABA}$ condition, and an improvement of $75.03 \%$ with $85 \mathrm{mM} \mathrm{NaCl}$ (Figure 8B). Results of dry weight measurements confirmed the conclusion that GmERF135 could promote plant growth under ABA and $\mathrm{NaCl}$ conditions.

\section{DISCUSSION}

Abscisic acid is well-known to be involved in responses to multiple stresses, such as drought, salt, and cold, and to induce expression of stress-related genes in plants (Leung et al., 1997; 


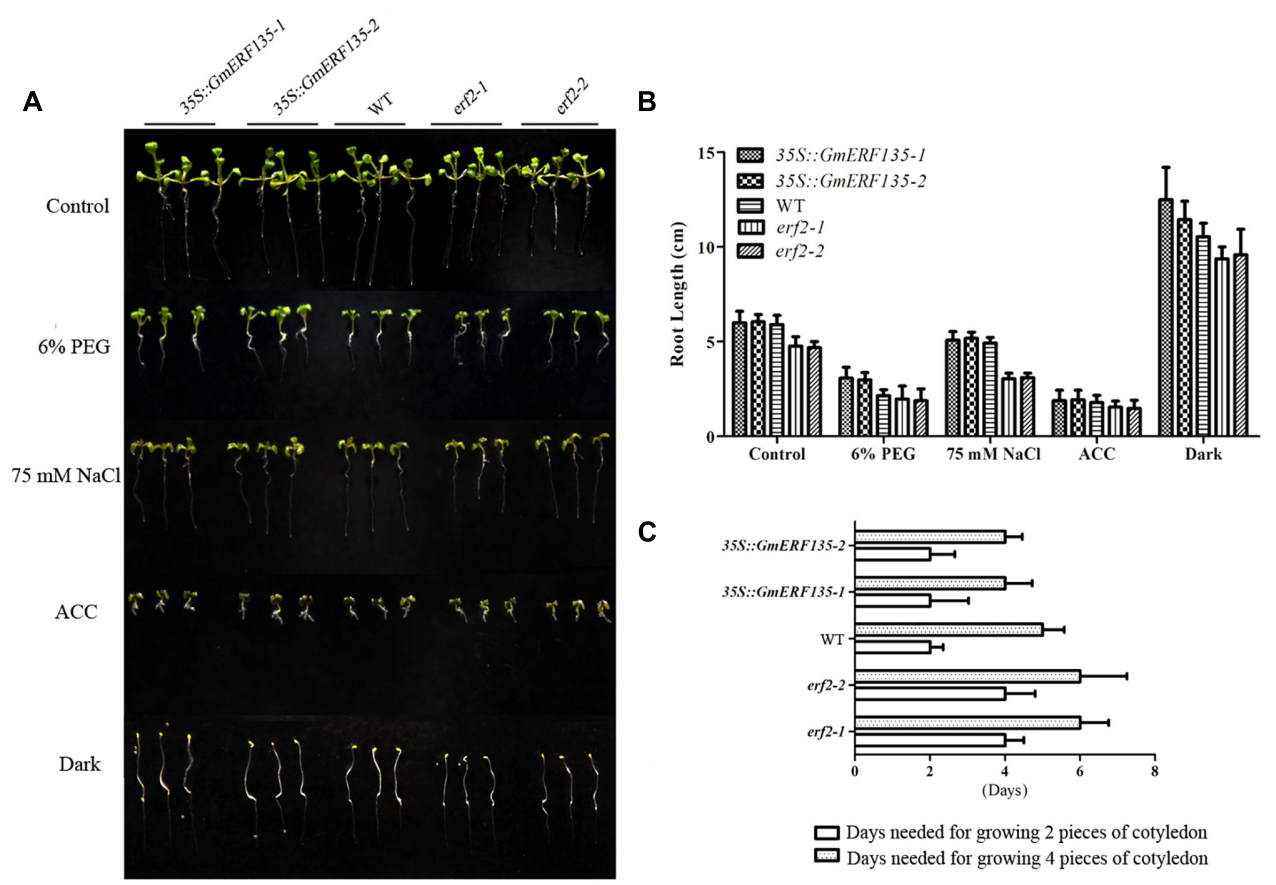

FIGURE 7 | GmERF135 transgenic Arabidopsis lines improved cotyledon growth under the NaCl and PEG conditions. (A) Phenotype of GmERF135 transgenic Arabidopsis lines under various stresses. Three-day-old GmERF135 transgenic Arabidopsis lines were transferred to 1/2 MS medium with 6\% PEG, $75 \mathrm{mM} \mathrm{NaCl,}$ ACC, or grown in darkness. (B) Average root lengths of WT, erf2, and 35S::GmERF135 lines. Three-day-old seedlings were treated with various stresses for 3 days and the data were shown as the means \pm SD of three biological replicates (at least 30 individuals per line). (C) Days needed for growing cotyledon. Statistic data were recorded every $12 \mathrm{~h}$. Three biological replicates were performed.

A

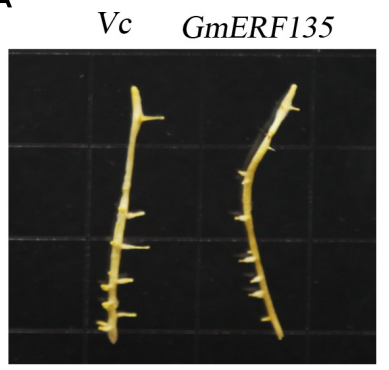

MS0

B

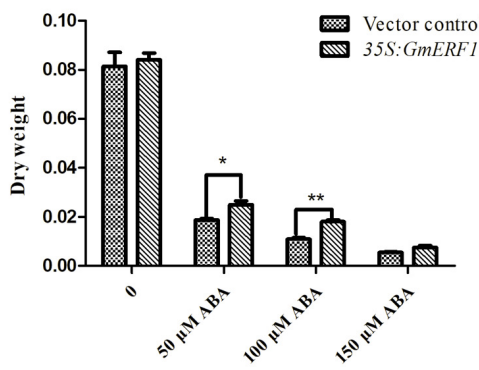

$V c$

GmERF 135

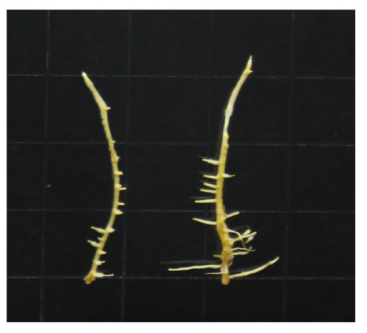

$100 \mu \mathrm{M}$ ABA
Vc GmERF135

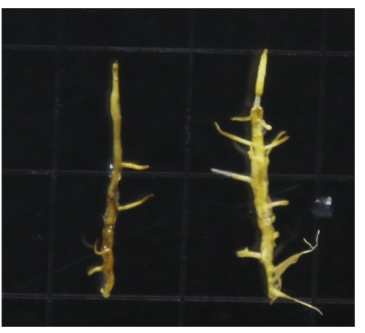

$85 \mathrm{mM} \mathrm{NaCl}$

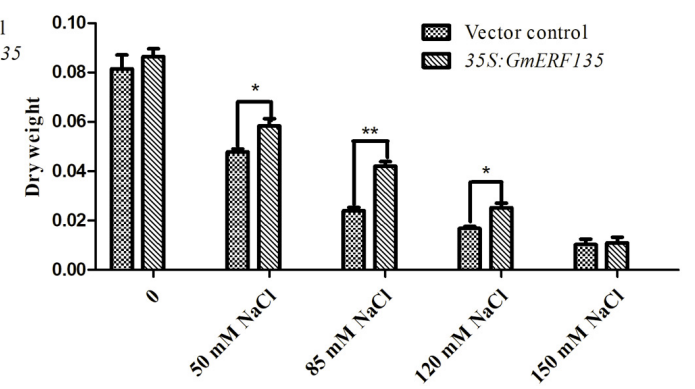

FIGURE 8| GmERF135 transgenic soybean hairy roots enhanced tolerance to ABA and salt. (A) Phenotypes of WT and GmERF135 transgenic soybean hairy roots grown on MS medium with different concentrations of ABA and NaCl. Vc for Vector control. (B) Dry weight of WT and transgenic soybean hairy roots after 2 weeks of treatment. Data are shown as means \pm SD of three biological experiments. Student's $t$ test was used for statistical analysis and asterisks indicate significant differences from the vector control at ${ }^{*} P<0.05$ and ${ }^{* *} P<0.01$. 
Rohde et al., 2000; Rook et al., 2001; Xu et al., 2007). The interaction of the ABA and ET signal pathway is extremely complex and intricate. AREB/ABFs, members of the bZIP family of transcription factors, participate in the ABA signal pathway which can specifically bind to ABRE cis-element to modulate the expression of downstream target genes (Bonetta and McCourt, 1998; Leung and Giraudat, 1998; Li et al., 2012). Fujita et al. (2005) observed that AREB1 can act as a transcription activator in ABRE-dependent ABA signaling, which enhances drought tolerance in Arabidopsis. Overexpression of the AREB1 gene in Arachis hypogaea could enhance drought tolerance by modulating ROS scavenging and maintaining content (Li et al., 2013). Phosphorylated AREB/ABFs may bind to the ABRE ciselement of GmERF135 promotor region to activate its transcription level (Fujita et al., 2013), which could activate or repress the transcription of targeted genes. In our study, analysis of the GmERF135 promoter region showed that three ABREs were located at the promoter region, which may be binding sites of AREB/ABFs to affect the transcript of GmERF135. GmERF135 has a conversed AP2/ERF domain, which could specifically bind to GCC-box and/or dehydrationresponsive element/C-repeat (DRE/CRT) cis-acting elements to modulate the expression of pathogenesis- and abiotic stressesrelated genes (Hao et al., 1998; Chakravarthy et al., 2003; Zarei et al., 2011).

Previous studies showed there is an antagonistic interaction between $\mathrm{ABA}$ and JA-ET signaling pathways ABA for disease response (Beaudoin et al., 2000; Anderson et al., 2004; MartinRodriguez et al., 2011). A recent study showed that SlAREB1 could enhance the expression of ethylene biosynthetic genes $A C S$ and $A C O$ in tomato fruits, which are the two key genes of ET synthesis (Mou et al., 2018). In our study, qRT-PCR analysis showed that the transcription of both AtACO4 and AtACS2 increased (Supplementary Figure S2) in transgenic Arabidopsis lines, which suggested acceleration of ET production. ET acceleration could trigger a series of reactions of ET and activate the transcript of ERF responsive genes (Guo and Ecker, 2004). At the same time, the transcript levels of $A B A 1$ and $A B A 2$, key factors of ABA synthesis, were also upregulated after overexpressing GmERF135 in Arabidopsis lines. These results suggested that GmERF135 may participate in both ET and ABA signaling pathways, and the regulation between the two signaling pathways needs further research.

Except for ABREs, other stress-related elements were also distributed in the promotor region of GmERF135, such as the MYBST1 core sequence, MBS, and ERE (Table 1). The MYBST1 core sequence and MBS elements have been demonstrated to be involved in drought, low temperature, salt, and ABA stress responses (Abe et al., 2003). qRT-PCR showed that GmERF135 is induced by multiple stresses, including drought, salt, low temperature, exogenous ET, SA, and JA. These changes may be caused by some corresponding cis-elements in promoter regions which can be bound by specific transcription factors (Huang et al., 2012; Buscaill and Rivas, 2014). These results suggest that soybean
ERF gene GmERF135 is a key factor which participates in multiple signaling pathways to regulate expression levels of stress-related genes.

\section{AUTHOR CONTRIBUTIONS}

Z-SX and Y-ZM coordinated the project, conceived and designed experiments, and edited the manuscript. M-JZ and L-JY conducted the bioinformatic work, performed experiments and wrote the first draft. JM revised the manuscript and figures. J-CZ conducted the bioinformatic work. Y-XW, J-HL, and J-DF contributed with valuable discussions. MC provided analytical tools and managed reagents. All authors have read and approved the final manuscript.

\section{FUNDING}

This research was supported by the National Natural Science Foundation of China (31871624), the National Transgenic Key Project of Ministry of Agriculture (2018ZX0800909B), and the Technological Innovation Projects of Modern Agriculture of Hebei Province (494-0402-YBN-RDC4).

\section{ACKNOWLEDGMENTS}

We thank Professor Tian-Fu Han for providing the Superroot culture of L. corniculatus, A. rhizogenes strain K599, and the binary vector pGFPGUSPlus. We also thank Dr. WenSheng Hou for plant material preparation, technical assistance, and soybean seeds.

\section{SUPPLEMENTARY MATERIAL}

The Supplementary Material for this article can be found online at: https://www.frontiersin.org/articles/10.3389/fpls.2019.00940/ full\#supplementary-material

FIGURE S1 | Expression patterns of candidate soybean ERFs in different organs. (A) Expression patterns of candidate soybean ERFs in different organs. Normalized expression data for the soybean ERF genes were collected from the SoyBase (http://www.soybase.org/). Expression (vertical coordinates) is in transcripts per million (TPM). (B) Semi-quantitative PCR of GmERF135 in different organs.

FIGURE S2 | Express levels of various stresses-related genes after treatment. Two-week-old WT and transgenic Arabidopsis lines were used to extract total RNA. GmActin/AtActin was used for normalization. Data were shown as the means \pm SDs of three experiments.

TABLE S1 | Specific primers of each gene for qRT-PCR. All the primers were designed via Primer Primer 5.0.

TABLE S2 | Classification of 160 soybean ERF genes. 


\section{REFERENCES}

Abe, H., Urao, T., Ito, T., Seki, M., Shinozaki, K., and Yamaguchi-Shinozaki, K. (2003). Arabidopsis AtMYC2 (bHLH) and AtMYB2 (MYB) function as transcriptional activators in abscisic acid signaling. Plant Cell 15, 63-78. doi: 10.1105/tpc.006130

Allen, M. D., Yamasaki, K., Ohme-Takagi, M., Tateno, M., and Suzuki, M. (1998). A novel mode of DNA recognition by a beta-sheet revealed by the solution structure of the GCC-box binding domain in complex with DNA. EMBO J. 17, 5484-5496. doi: 10.1093/emboj/17.18.5484

Anderson, J. P., Badruzsaufari, E., Schenk, P. M., Manners, J. M., Desmond, O. J., Ehlert, C., et al. (2004). Antagonistic interaction between abscisic acid and jasmonate-ethylene signaling pathways modulates defense gene expression and disease resistance in Arabidopsis. Plant Cell 16, 3460-3479. doi: 10.1105/tpc. 104.025833

Beaudoin, N., Serizet, C., Gosti, F., and Giraudat, J. (2000). Interactions between abscisic acid and ethylene signaling cascades. Plant Cell 12, 1103-1115. doi: $10.1105 /$ tpc.12.7.1103

Bechtold, N., and Pelletier, G. (1998). In planta agrobacterium-mediated transformation of adult Arabidopsis thaliana plants by vacuum infiltration. Methods Mol. Biol. 82, 259-266. doi: 10.1385/0-89603-391-0:259

Bonetta, D., and McCourt, P. (1998). Genetic analysis of ABA signal transduction pathways. Trends Plant Sci. 3, 231-235. doi: 10.1016/s1360-1385(98)01241-2

Buscaill, P., and Rivas, S. (2014). Transcriptional control of plant defence responses. Curr. Opin. Plant Biol. 20, 35-46. doi: 10.1016/j.pbi.2014.04.004

Chakravarthy, S., Tuori, R. P., D’Ascenzo, M. D., Fobert, P. R., Despres, C., and Martin, G. B. (2003). The tomato transcription factor Pti4 regulates defenserelated gene expression via GCC box and non-GCC box cis elements. Plant Cell 15, 3033-3050. doi: 10.1105/tpc.017574

Chen, L., Jiang, B., Wu, C., Sun, S., Hou, W., and Han, T. (2014). GmPRP2 promoter drives root-preferential expression in transgenic Arabidopsis and soybean hairy roots. BMC Plant Biol. 14:245. doi: 10.1186/s12870-014-0245-Z

Chen, M., Chory, J., and Fankhauser, C. (2004). Light signal transduction in higher plants. Annu. Rev. Genet. 38, 87-117. doi: 10.1146/annurev.genet.38.072902. 092259

Finkelstein, R. R., Gampala, S. S. L., and Rock, C. D. (2002). Abscisic acid signaling in seeds and seedlings. Plant Cell 14, S15-S45.

Fujita, Y., Fujita, M., Satoh, R., Maruyama, K., Parvez, M. M., Seki, M., et al. (2005). AREB1 is a transcription activator of novel ABRE-dependent ABA signaling that enhances drought stress tolerance in Arabidopsis. Plant Cell 17, 3470-3488. doi: $10.1105 /$ tpc.105.035659

Fujita, Y., Yoshida, T., and Yamaguchi-Shinozaki, K. (2013). Pivotal role of the AREB/ABF-SnRK2 pathway in ABRE-mediated transcription in response to osmotic stress in plants. Physiol. Plant. 147, 15-27. doi: 10.1111/j.1399-3054. 2012.01635. $\mathrm{x}$

Goodstein, D. M., Shu, S., Howson, R., Neupane, R., Hayes, R. D., Fazo, J., et al. (2012). Phytozome: a comparative platform for green plant genomics. Nucleic Acids Res. 40, D1178-D1186. doi: 10.1093/nar/gk r944

Guo, H., and Ecker, J. R. (2004). The ethylene signaling pathway: new insights. Curr. Opin. Plant Biol. 7, 40-49. doi: 10.1016/j.pbi.2003.11.011

Hao, D., Ohme-Takagi, M., and Sarai, A. (1998). Unique mode of GCC box recognition by the DNA-binding domain of ethylene-responsive elementbinding factor (ERF domain) in plant. J. Biol. Chem. 273, 26857-26861. doi: 10.1074/jbc.273.41.26857

He, G. H., Xu, J. Y., Wang, Y. X., Liu, J. M., Li, P. S., Chen, M., et al. (2016). Drought-responsive WRKY transcription factor genes TaWRKY1 and TaWRKY33 from wheat confer drought and/or heat resistance in Arabidopsis. BMC Plant Biol. 16:116. doi: 10.1186/s12870-016-0806-4

Huang, G. T., Ma, S. L., Bai, L. P., Zhang, L., Ma, H., Jia, P., et al. (2012). Signal transduction during cold, salt, and drought stresses in plants. Mol. Biol. Rep. 39, 969-987. doi: 10.1007/s11033-011-0823-1

Hubbard, K. E., Nishimura, N., Hitomi, K., Getzoff, E. D., and Schroeder, J. I. (2010). Early abscisic acid signal transduction mechanisms: newly discovered components and newly emerging questions. Genes Dev. 24, 1695-1708. doi: 10.1101/gad.1953910

Jin, Y., Pan, W. Y., Zheng, X. F., Cheng, X., Liu, M. M., Ma, H., et al. (2018). OsERF101, an ERF family transcription factor, regulates drought stress response in reproductive tissues. Plant Mol. Biol. 98, 51-65. doi: 10.1007/ s11103-018-0762-5

Kim, J.-G., Stork, W., and Mudgett, M. B. (2013). Xanthomonas type III effector XopD desumoylates tomato transcription factor SIERF4 to suppress ethylene responses and promote pathogen growth. Cell Host Microbe 13, 143-154. doi: 10.1016/j.chom.2013.01.006

Klay, I., Gouia, S., Lu, M., Mila, I., Khoudi, H., Bernadac, A., et al. (2018). Ethylene response factors (ERF) are differentially regulated by different abiotic stress types in tomato plants. Plant Sci. 274, 137-145. doi: 10.1016/j.plantsci.2018. 05.023

Le, D. T., Nishiyama, R., Watanabe, Y., Mochida, K., Yamaguchi-Shinozaki, K., Shinozaki, K., et al. (2011). Genome-wide expression profiling of soybean twocomponent system genes in soybean root and shoot tissues under dehydration stress. DNA Res. 18, 17-29. doi: 10.1093/dnares/dsq032

Lescot, M., Dehais, P., Thijs, G., Marchal, K., Moreau, Y., Van de Peer, Y., et al. (2002). PlantCARE, a database of plant cis-acting regulatory elements and a portal to tools for in silico analysis of promoter sequences. Nucleic Acids Res. 30, 325-327. doi: 10.1093/nar/30.1.325

Leung, J., and Giraudat, J. (1998). Cloning genes of Arabidopsis thaliana by chromosome walking. Methods Mol. Biol. 82, 277-303. doi: 10.1385/0-89603391-0:277

Leung, J., Merlot, S., and Giraudat, J. (1997). The Arabidopsis ABSCISIC ACID-INSENSITIVE2 (ABI2) and ABI1 genes encode homologous protein phosphatases 2C involved in abscisic acid signal transduction. Plant Cell 9, 759-771. doi: 10.1105/tpc.9.5.759

Li, W., Cui, X., Meng, Z. L., Huang, X. H., Xie, Q., Wu, H., et al. (2012). Transcriptional regulation of Arabidopsis MIR168a and argonaute1 homeostasis in abscisic acid and abiotic stress responses. Plant Physiol. 158, 1279-1292. doi: 10.1104/pp.111.188789

Li, X. Y., Liu, X., Yao, Y., Li, Y. H., Liu, S., He, C. Y., et al. (2013). Overexpression of Arachis hypogaea AREB1 gene enhances drought tolerance by modulating ROS scavenging and maintaining endogenous ABA content. Int. J. Mol. Sci. 14, 12827-12842. doi: 10.3390/ijms140612827

Liu, D. F., Chen, X. J., Liu, J. Q., Ye, J. C., and Guo, Z. J. (2012). The rice ERF transcription factor OsERF922 negatively regulates resistance to Magnaporthe oryzae and salt tolerance. J. Exp. Bot. 63, 3899-3911. doi: 10.1093/jxb/ers079

Liu, P., Xu, Z. S., Pan-Pan, L., Hu, D., Chen, M., Li, L. C., et al. (2013). A wheat PI4K gene whose product possesses threonine autophophorylation activity confers tolerance to drought and salt in Arabidopsis. J. Exp. Bot. 64, 2915-2927. doi: 10.1093/jxb/ert133

Liu, Q., Kasuga, M., Sakuma, Y., Abe, H., Miura, S., Yamaguchi-Shinozaki, K., et al. (1998). Two transcription factors, DREB1 and DREB2, with an EREBP/AP2 DNA binding domain separate two cellular signal transduction pathways in drought- and low-temperature-responsive gene expression, respectively, in Arabidopsis. Plant Cell 10, 1391-1406. doi: 10.1105/tpc.10.8.1391

Ma, Y., Szostkiewicz, I., Korte, A., Moes, D., Yang, Y., Christmann, A., et al. (2009). Regulators of PP2C phosphatase activity function as abscisic acid sensors. Science 324, 1064-1068. doi: 10.1126/science.1172408

Martin-Rodriguez, J. A., Leon-Morcillo, R., Vierheilig, H., Ocampo, J. A., LudwigMuller, J., and Garcia-Garrido, J. M. (2011). Ethylene-dependent/ethyleneindependent $\mathrm{ABA}$ regulation of tomato plants colonized by arbuscular mycorrhiza fungi. New Phytol. 190, 193-205. doi: 10.1111/j.1469-8137.2010. 03610.x

Mou, W., Li, D., Luo, Z., Li, L., Mao, L., and Ying, T. (2018). SlAREB1 transcriptional activation of NOR is involved in abscisic acid-modulated ethylene biosynthesis during tomato fruit ripening. Plant Sci. 276, 239-249. doi: 10.1016/j.plantsci.2018.07.015

Okamuro, J. K., Caster, B., Villarroel, R., Van Montagu, M., and Jofuku, K. D. (1997). The AP2 domain of APETALA2 defines a large new family of DNA binding proteins in Arabidopsis. Proc. Natl. Acad. Sci. U.S.A. 94, 7076-7081. doi: 10.1073/pnas.94.13.7076

Pandey, S., Chen, J. G., Jones, A. M., and Assmann, S. M. (2006). G-protein complex mutants are hypersensitive to abscisic acid regulation of germination and postgermination development. Plant Physiol. 141, 243-256. doi: 10.1104/ pp.106.079038

Pandey, S., Nelson, D. C., and Assmann, S. M. (2009). Two novel GPCR-type $\mathrm{G}$ proteins are abscisic acid receptors in Arabidopsis. Cell 136, 136-148. doi: 10.1016/j.cell.2008.12.026 
Park, S. Y., Fung, P., Nishimura, N., Jensen, D. R., Fujii, H., Zhao, Y., et al. (2009). Abscisic acid inhibits type $2 \mathrm{C}$ protein phosphatases via the PYR/PYL family of START proteins. Science 324, 1068-1071. doi: 10.1126/science.117 3041

Rattanakon, S., Ghan, R., Gambetta, G. A., Deluc, L. G., Schlauch, K. A., and Cramer, G. R. (2016). Abscisic acid transcriptomic signaling varies with grapevine organ. BMC Plant Biol. 16:72. doi: 10.1186/s12870-0160763-y

Rohde, A., Kurup, S., and Holdsworth, M. (2000). ABI3 emerges from the seed. Trends Plant Sci. 5, 418-419. doi: 10.1016/s1360-1385(00)01 736-2

Rook, F., Corke, F., Card, R., Munz, G., Smith, C., and Bevan, M. W. (2001). Impaired sucrose-induction mutants reveal the modulation of sugar-induced starch biosynthetic gene expression by abscisic acid signalling. Plant J. 26, 421-433. doi: 10.1046/j.1365-313x.2001. 2641043. $\mathrm{x}$

Ruggiero, B., Koiwa, H., Manabe, Y., Quist, T. M., Inan, G., Saccardo, F., et al. (2004). Uncoupling the effects of abscisic acid on plant growth and water relations. Analysis of sto1/nced3, an abscisic acid-deficient but salt stresstolerant mutant in Arabidopsis. Plant Physiol. 136, 3134-3147. doi: 10.1104/ pp.104.046169

Santiago, J., Rodrigues, A., Saez, A., Rubio, S., Antoni, R., Dupeux, F., et al. (2009). Modulation of drought resistance by the abscisic acid receptor PYL5 through inhibition of clade A PP2Cs. Plant J. 60, 575-588. doi: 10.1111/j.1365-313X. 2009.03981.x

Scott, A., Wyatt, S., Tsou, P. L., Robertson, D., and Allen, N. S. (1999). Model system for plant cell biology: GFP imaging in living onion epidermal cells. Biotechniques 26, 1125, 1128-1132. doi: 10.2144/9926 6 st04

Shen, Y. Y., Wang, X. F., Wu, F. Q., Du, S. Y., Cao, Z., Shang, Y., et al. (2006). The Mg-chelatase $\mathrm{H}$ subunit is an abscisic acid receptor. Nature 443, 823-826. doi: $10.1038 /$ nature 05176

Wang, X., Guo, C., Peng, J., Li, C., Wan, F., Zhang, S., et al. (2018). ABREBINDING FACTORS play a role in the feedback regulation of ABA signaling by mediating rapid ABA induction of ABA co-receptor genes. New Phytol. 221, 341-355. doi: 10.1111/nph.15345
Xu, Z. S., Chen, M., Li, L. C., and Ma, Y. Z. (2008). Functions of the ERF transcription factor family in plants. Bot. Botanique 86, 969-977. doi: 10.1139/ b08-041

Xu, Z. S., Chen, M., Li, L. C., and Ma, Y. Z. (2011). Functions and application of the AP2/ERF transcription factor family in crop improvement. J. Integr. Plant Biol. 53, 570-585. doi: 10.1111/j.1744-7909.2011.01062.x

Xu, Z. S., Xia, L. Q., Chen, M., Cheng, X. G., Zhang, R. Y., Li, L. C., et al. (2007). Isolation and molecular characterization of the Triticum aestivum L. ethyleneresponsive factor 1 (TaERF1) that increases multiple stress tolerance. Plant Mol. Biol. 65, 719-732. doi: 10.1007/s11103-007-9237-9

Yamaguchi-Shinozaki, K., and Shinozaki, K. (1994). A novel cis-acting element in an Arabidopsis gene is involved in responsiveness to drought, low-temperature, or high-salt stress. Plant Cell 6, 251-264. doi: 10.1105/tpc.6.2.251

Yang, Y., and Guo, Y. (2018). Unraveling salt stress signaling in plants. J. Integr. Plant Biol. 60, 796-804. doi: 10.1111/jipb.12689

Yoshida, T., Fujita, Y., Maruyama, K., Mogami, J., Todaka, D., Shinozaki, K., et al. (2015). Four Arabidopsis AREB/ABF transcription factors function predominantly in gene expression downstream of SnRK2 kinases in abscisic acid signalling in response to osmotic stress. Plant Cell Environ. 38, 35-49. doi: $10.1111 /$ pce. 12351

Zarei, A., Korbes, A. P., Younessi, P., Montiel, G., Champion, A., and Memelink, J. (2011). Two GCC boxes and AP2/ERF-domain transcription factor ORA59 in jasmonate/ethylene-mediated activation of the PDF1.2 promoter in Arabidopsis. Plant Mol. Biol. 75, 321-331. doi: 10.1007/s11103-010-9728-y

Conflict of Interest Statement: The authors declare that the research was conducted in the absence of any commercial or financial relationships that could be construed as a potential conflict of interest.

Copyright (C) 2019 Zhao, Yin, Ma, Zheng, Wang, Lan, Fu, Chen, Xu and Ma. This is an open-access article distributed under the terms of the Creative Commons Attribution License (CC BY). The use, distribution or reproduction in other forums is permitted, provided the original author(s) and the copyright owner(s) are credited and that the original publication in this journal is cited, in accordance with accepted academic practice. No use, distribution or reproduction is permitted which does not comply with these terms. 